\title{
REFERENCES
}

COOPER, D. W., AND RENDEL, J. 1968. Incomplete family data, selection and population structure for bovine transferins and blood groups. Heredity, 23, 49-66.

CRow, J. F. 1945. A chart of the $\mathrm{X}^{2}$ and $t$ distributions. F. Am. Stat. Assoc., 40, 376.

FISHER, R. A. 1960. The Design of Experiments, 7th Ed. Hafner Publishing Co. Inc., New York.

NEIMANN-SORENSEN, A., AND ROBERTSON, A. 1961. The association between blood groups and several production characteristics in three Danish cattle breeds. Acta Agric. Scand., 11, 163-196.

PEARSON, E. S., AND haRtLey, H. o. 1958. Biometrika Tables for Statisticians, Vol. I. Cambridge University Press.

\section{STABILISING SELECTION IN THE ABSENCE OF DOMINANCE: AN ADDITIONAL NOTE}

\author{
M. J. KEARSEY and J. S. GALE \\ Department of Genetics, University of Birmingham
}

\section{InTRODUGTION}

IN an earlier paper (Gale and Kearsey, 1968) it was shown, for a 2-locus model, that stabilising selection can lead to stable equilibria, even if the genes act in a purely additive manner in regard to their effect on the primary character under selection. The necessary condition for such equilibria to occur, given an additive system, is that the effect of gene substitutions on the primary character shall differ from one locus to the other. If this disparity between loci is small, equilibria will result only if the loci are closely linked; the greater the disparity, the looser need be the linkage. If the disparity is very large, stable equilibria will obtain even in the absence of linkage. Results strongly suggest that in situations where stable equilibria exist, they will usually be attained, irrespective of the initial gametic frequencies.

It is natural to enquire whether these results are applicable to more realistic situations, where the primary character is under the control of many loci. In view of the many parameters required to describe such situations, a systematic investigation of this problem would be a formidable undertaking and seems scarcely justified at present, since the values of such parameters are known only for a few special cases. We shall therefore confine ourselves to describing the results obtained by computer simulation of a few special situations, using a 3-locus deterministic model. It will be shown that, at least for the cases considered, results on the 3-locus model do not differ in any essential way from those obtained on the 2-locus model considered earlier.

\section{Models of STABILISING SELEction}

A wide variety of models representing the change of fitness with phenotype under stabilising selection have been proposed at various times. Unfortunately there is little evidence which would enable us to decide which models are really appropriate. In the present paper, we shall consider 2 models of the same general type but with different intensities of 
selection. In the first (model A), individuals at the midpoint of the phenotypic range have fitness unity. Fitness falls off linearly with phenotype on either side of the optimum, reaching zero for the 2 most extreme phenotypes possible, that is

$$
\text { Fitness }=1-\left|\frac{P-M}{E-M}\right|
$$

where $P$ is the phenotype under consideration,

$M$ is the mid-phenotype,

$E$ is either of the two extreme phenotypes.

The second model (model B) differs from model A in that fitness, while still falling off linearly with phenotype, reaches zero when the phenotype is halfway between mid-phenotype and extreme phenotype; that is, if we put

we then have

$$
X=1-2\left|\frac{P-M}{E-M}\right|
$$

$$
\begin{aligned}
\text { Fitness } & =X \text { if } X \geqq 0 \\
& =0 \text { otherwise }
\end{aligned}
$$

Model B receives some support from studies of fitness in cage populations of Drosophila melanogaster (Barnes and Kearsey, personal communication). Model A was introduced purely for purposes of comparison.

\section{Results of simulations}

Apart from one case described below, all runs were started with gametic frequencies as follows:

$\begin{array}{ll}A B C & 0.7300 \\ A B c & 0.0795 \\ A b C & 0.0810 \\ A b c & 0.0090 \\ a B C & 0.0815 \\ a B c & 0.0095 \\ a b C & 0.0085 \\ a b c & 0.0010\end{array}$

Runs were continued until stable equilibrium values could be determined to the fourth decimal place or alternatively until it was clear that no such equilibrium existed.

To facilitate description of the various situations investigated, phenotypes. on the primary scale will be represented as

Genotype Phenotype Genotype Phenotype Genotype Phenotype

$\begin{array}{cccccc}a a & 0 & b b & 0 & c c & 0 \\ A a & k & B b & l & C c & m \\ A A & 2 k & B B & 2 l & C C & 2 m\end{array}$

with no epistasis on the primary scale. Recombination fractions will be denoted $X, Y$ where $X$ is the recombination fraction between $A / a$ and $B / b$, 
and $Y$ the recombination fraction between $B / b$ and $C / c$; for simplicity it was assumed that there was no interference.

In presenting the results, the term equilibrium will be used as short for stable equilibrium.

Consider first model B. With

$$
k=1 \cdot 0, l=1 \cdot 8, m=0 \cdot 48, X=0 \cdot 1, Y=0 \cdot 1
$$

equilibrium was obtained. Values of $l, m$ were then varied independently in steps of 0.01 . With $l=1.79$ or less, or with $m=0.49$ or more, no equilibrium was possible. Thus a fairly striking difference between the effects of gene substitutions at the 3 loci was necessary.

We may now consider the effect of linkage. With

$$
X=0.5, r=0.5
$$

equilibrium was obtained when

$$
k=1 \cdot 0, l=1 \cdot 79, m=0 \cdot 17 .
$$

No equilibrium results if $l=1.78$ or less, or if $m=0.18$ or more. Thus in the absence of linkage, the discrepancy between gene effects at the 3 loci must be even more extreme than in case (1).

It should be emphasised that, if we put

$$
k=1 \cdot 8, l=1 \cdot 0, m=0 \cdot 48, X=0 \cdot 1, Y=0 \cdot 1
$$

equilibrium values are not the same as in case (1), despite the fact that we have merely interchanged the values of $k$ and $l$. This is hardly surprising, since in case (3) the most discrepant loci are those showing the greatest amount of recombination, in contrast to the situation in case (1).

With milder selection, the discrepancy between loci must be increased if equilibrium is to result. Thus with model $\mathrm{A}$, and

$$
k=1 \cdot 0, l=1 \cdot 8, m=0 \cdot 25, X=0 \cdot 1, Y=0 \cdot 1
$$

equilibrium was obtained, but if $m=0.26$ or more, no equilibrium was possible.

Finally, we may consider the possible importance of the initial gametic frequencies. In particular, we may ask, for some of the situations where no equilibrium was obtained, whether an equilibrium would result if initial frequencies were rather different from those used heretofore.

Consider the case

$$
k=1 \cdot 0, l=1 \cdot 8, m=0 \cdot 49, X=0 \cdot 1, r=0 \cdot 1
$$

with selection according to model B. This case is not very different from (1) and hence, if equilibrium were possible, given appropriate initial frequencies, equilibrium frequencies should be close to those found in case (1). Hence, if we start case (5) with the case (1) equilibrium values, we should be able to detect such an hypothesised equilibrium. In fact, however, no equilibrium is found under such circumstances. This supports the conclusion, tentatively drawn for the 2-locus model, that the final result is not very dependent on initial frequencies.

Equilibrium frequencies for cases (1) to (4) are given in table 1. 


\section{TABLE 1}

Gametic frequencies at equilibrium

Case $A B C$ or $a b c A B c$ or $a b C A b C$ or $a B c A b c$ or $a B C$

$\begin{array}{lllll}\text { (1) } & 0.0073 & 0.0353 & 0.3821 & 0.0753 \\ (2) & 0.0944 & 0.1034 & 0.1540 & 0.1482 \\ (3) & 0.0252 & 0.0096 & 0.3898 & 0.0754 \\ (4) & 0.0276 & 0.0474 & 0.2614 & 0.1636\end{array}$

\section{Summary}

1. A number of cases of stabilising selection, in which the primary character is controlled by 3 loci with additive gene action, have been investigated, using computer simulations.

2. Stable equilibria were obtained in those cases where the effects on the primary character were markedly different from one locus to another. When these differences were sufficiently large, equilibria resulted, even when the loci were unlinked.

3. The magnitude of the difference between loci which is necessary for stable equilibrium to occur falls off with intensity of linkage and intensity of selection.

4. Results suggest that the attainment or non-attainment of equilibrium is not very dependent on initial gametic frequencies.

5. These results agree with those previously obtained for a 2-locus model.

Acknowledgments.-We should like to thank Mr P. G. Walling, of the University of Birmingham Computer Services, for assistance in the running of the computer programme. This work was supported by a grant from the Agricultural Research Council.

\section{REFERENGES}

GALE, J. S., AND KEARSEY, M. J. 1968. Stable equilibria under stabilising selection in the absence of dominance. Heredity 23, 553-561. 